\title{
Combined Effect of Non-Uniform Temperature Gradient and Magnetic Field on Bénard-Marangoni Convection with a Constant Heat Flux
}

\author{
Siti Suzilliana Putri Mohamed Isa ${ }^{1}$, Norihan Md. Arifin ${ }^{*}, 1$, Roslinda Mohd Nazar² and Mohd Noor Saad ${ }^{1}$ \\ ${ }^{I}$ Department of Mathematics and Institute for Mathematical Research, Universiti Putra Malaysia, 43400 UPM Serdang, \\ Selangor, Malaysia \\ ${ }^{2}$ School of Mathematical Sciences, Faculty of Science and Technology, Universiti Kebangsaan Malaysia, 43600 UKM \\ Bangi, Selangor, Malaysia
}

\begin{abstract}
This paper studied the problem of Bénard-Marangoni convection in a horizontal fluid layer heated from below with non-uniform temperature gradient under magnetic field. A linear stability analysis is performed to undertake a detail investigation. The eigenvalues are obtained for both adiabatic boundaries. The influence of various parameters on the onset of convection has been analyzed. Six non-uniform basic temperature profiles are considered and some general conclusions about their destabilizing effects are presented.
\end{abstract}

Keywords: Bénard-Marangoni Convection, magnetic field, non-uniform temperature.

\section{INTRODUCTION}

Convection has been the subject of many investigations due to the related engineering applications such as crystal growth, weld penetration and in coating process. In recent years, there has been a great increase of interest in the theory and modeling of material processing in the microgravity environment. A theory and modeling of material processing in the laboratory may include the mechanism of suppressing free convection driven by both buoyancy and surface tension forces. Rayleigh's paper is the pioneering work for almost all modern theories of convection. Rayleigh [1] showed that Bénard convection, which is caused by buoyancy effects, will occur when the Rayleigh number exceeds a critical value. The first theoretical study of convection caused by the thermocapillary effects was done by Pearson [2]. He analyzed Marangoni convection in the case of infinite fluid layer, a non-deformable free surface and zero gravity for noslip boundary conditions at the bottom. He showed that Marangoni convection occurs when the Marangoni number exceeds a critical value. The analysis of Bénard-Marangoni convection induced by thermal buoyancy and surface tension was treated by Nield [3]. He studied the Bénard-Marangoni convective instability in a planar horizontal fluid layer with non-deformable free surface. He showed that for steady convection, the two destabilizing mechanism reinforce one another.

The above investigations are limited to a uniform basic temperature gradient. Vidal and Acrivos [4], Debler and Wolf [5] and Nield [6] studied the effect of a non-uniform temperature gradient on the onset of Marangoni convection. Rudraiah [7] and Friedrich and Rudraiah [8] have examined

\footnotetext{
*Address correspondence to this author at the Department of Mathematics and Institute for Mathematical Research, Universiti Putra Malaysia, 43400 UPM Serdang, Selangor, Malaysia; Tel: 603-89466850;

Fax:603-89437958; E-mail: norihan@math.upm.edu.my
}

the combined effect of rotation and non-uniform basic temperature gradient on Marangoni convection. The effects of non-uniform temperature gradient on Bénard-Marangoni convection were studied by Lebon and Cloot [9] and the combined effects of non-uniform temperature gradient and Coriolis force (due to rotation) on the Bénard-Marangoni convection were analyzed by Rudraiah and Ramachandramurthy [10]. The results of [8-10] proved that the Coriolis force and suitable non-uniform temperature gradient suppress convection. Chiang [11] studied the effect of nonuniform temperature gradient on the onset of stationary and oscillatory Bénard-Marangoni convection. He showed that the effect of crispation at a deformable upper free surface is destabilizing factor but the conductive state of non-steady conditions within the fluid layer does play a stabilizing state. Very recently, Idris et al. [12] studied the effect of nonuniform basic temperature gradient on Bénard-Marangoni convection in micropolar fluid. They showed that the onset of convection can be delayed by the application of a cubic basic state temperature profile.

The effect of magnetic field on the onset of steady buoyancy-driven convection subject to a uniform basic temperature gradient was treated by Chandrasekhar [13] who showed that the effect of magnetic field is to increase the critical value of Rayleigh number and hence to have a stabilizing effect on the layer. The effect of magnetic field on the onset of steady Benard-Marangoni convection in a fluid layer with a nondeformable free surface was first analyzed by Nield [14]. He found that the critical Marangoni number monotonically increased as the strength of vertical magnetic field increased. The combined effects of nonuniform temperature gradient and magnetic field on Marangoni convection have been studied by Rudraiah et al. [15]. Using the single-term Galerkin expansion procedure, they showed that a suitable non-uniform temperature gradient and magnetic field suppress Marangoni convection. 
Rudraiah et al. [16] have further studied about the effect of magnetic field and non-uniform temperature gradient on Bénard-Marangoni convection. They analyzed the convection in the case of isothermal at the bottom boundary of the fluid layer. Later, Chan and Chen [17] extended the work of Rudraiah et al. [16] to include a deformable free surface. They found that crispation at a deformable upper free surface makes a more unstable system.

The instabilities of Bénard-Marangoni convection have been investigated in many previous works. However, little research has been conducted in the case of a constant heat flux at a lower boundary in which no perturbation in the heat flux is allowed. Motivated by these previous works, we aim to study the onset of Bénard-Marangoni convection in a horizontal fluid layer under externally imposed uniform magnetic field including the effect of non-uniform temperature gradient with a constant heat flux at a lower boundary, where no perturbation in the heat flux is allowed (insulating case). The linear stability theory is applied and the resulting eigenvalue problem is solved using the singleterm Galerkin expansion procedure.

\section{MATHEMATICAL FORMULATION}

Consider a horizontal fluid layer of depth $d$ with a free upper surface heated from below and subjected to a uniform vertical temperature gradient. The fluid layer is bound below by a rigid, electrically and thermally-perfect insulating wall and bounded above by a free surface. This free surface is subject to a constant heat flux. The interface has a surface tension, $\tau$, which is assumed to be a linear function of the temperature as follows:

$\tau=\tau_{0}-\gamma\left(T-T_{0}\right)$,

where $\tau_{0}$ is the value of $\tau$ at temperature $T_{0}$ and the constant $\gamma$ is positive for most fluids. We use Cartesian coordinates with two horizontal $x$ - and $y$-axes located at the lower solid boundary and a positive $z$ - axis is directed towards the free surface. The magnetic field, $\mathbf{H}$ acts in the $z$ - direction.

In the basic state, the velocity $\mathbf{U}$, the temperature $T$ and the magnetic field $\mathbf{H}$ have the following solutions

$$
\mathbf{U}=0, \mathbf{H}=H \hat{\mathbf{k}},-\frac{d}{\Delta T} \frac{\mathrm{d} T_{0}}{\mathrm{~d} z}=f(z),
$$

where $\hat{\mathrm{k}}$ is the unit vector in the $z$ - direction and $f(z)$ is a nondimensional temperature gradient satisfying the condition

$$
\int_{0}^{1} f(z) \mathrm{d} z=1 .
$$

Subject to the Boussinesq approximation, the governing equations for an incompressible, electrically conducting fluid in the presence of a magnetic field are expressed as follows:

Continuity equation:

$\nabla \cdot \mathbf{U}=0$,
Momentum equation:

$$
\left(\frac{\partial}{\partial \mathrm{t}}+\mathbf{U} \cdot \nabla\right) \mathbf{U}=-\frac{1}{\rho} \nabla \Pi+v \nabla^{2} \mathbf{U}+\frac{\rho}{\rho_{0}} \mathbf{g}+\frac{\mu}{4 \pi \rho_{0}}(\mathbf{H} \cdot \nabla) \mathbf{H},
$$

Energy equation:

$\left(\frac{\partial}{\partial \mathrm{t}}+\mathbf{U} \cdot \nabla\right) T=\kappa \nabla^{2} T$

Magnetic field equations:

$\nabla \cdot \mathbf{H}=0$,

$\left(\frac{\partial}{\partial t}+\mathbf{U} . \nabla\right) \mathbf{H}=(\mathbf{H} . \nabla) \mathbf{U}+\eta \nabla^{2} \mathbf{H}$,

where $\mathbf{U}, T, \mathbf{H}, \rho, \mathbf{g}, v, \kappa$ and $\eta$ denote the velocity, temperature, magnetic field, density, acceleration due to gravity, kinematic viscosity, thermal diffusivity and electrical resistivity, respectively. Magnetic pressure can be written as $\Pi=p+\mu|\mathbf{H}|^{2} / 8 \pi$ where $p$ is the fluid pressure and $\mu$ is the magnetic permeability. When motion occurs, the upper free surface of the layer will be deformable with its position at $z=d+f(x, y, t)$. At the free surface, we have the usual kinematic condition together with the conditions of continuity for the normal and tangential stresses. The temperature obeys the Newton's law of cooling, $k \partial T / \partial \mathbf{n}=h\left(T-T_{\infty}\right)$, where $k$ and $h$ are the thermal conductivity of the fluid and the heat transfer coefficient between the free surface and the air, respectively, and $\mathbf{n}$ is the outward unit normal to the free surface. The boundary conditions at the bottom wall, $z=0$, are no-slip and conducting to the temperature perturbations.

To simplify the analysis, it is convenient to write the governing equations and the boundary conditions in a dimensionless form. In the dimensionless formulation, scales for length, velocity, time, magnetic field and temperature gradient are taken to be $d, \kappa / d, d^{2} / v, H_{0}$ and $\Delta T$ respectively. Furthermore, five dimensionless groups appearing in the problem are the Rayleigh number $R=g \alpha \Delta T d^{3} / v \kappa$, Marangoni number $M=\gamma \Delta T d / \rho_{0} \kappa v$, Prandtl number $P_{r}=v / \kappa$, magnetic Prandtl number $P_{m}=\eta / \kappa \quad$ and the Chandrasekhar number $Q=\mu H^{2} d^{2} / 4 \pi \rho_{0} v \eta$. We study the linear stability of the basic state by seeking perturbed solutions for any quantity $\Phi(x, y, z, t)$ in terms of normal modes in the form

$\Phi(x, y, z, t)=\Phi_{0}(x, y, z)+\phi(z) \exp \left[i\left(\alpha_{x} x+\alpha_{y} y\right)+s t\right]$,

where $\Phi_{0}$ is the value of $\Phi$ in the basic state, $a=\left(\alpha_{x}^{2}+\alpha_{y}^{2}\right)^{1 / 2}$ is the total horizontal wave number of the disturbance and $s$ is a complex growth rate with a real part representing the growth rate of the instability and the imaginary part representing its frequency. At marginal 
stability, the growth rate $s$ of perturbation is zero and the real part of $s, \Re(s)>0$ represents unstable modes while $\Re(s)<0$ represents stable modes. Substituting equation (9) into equations (4) - (8) and neglecting terms of the second and higher orders in the perturbations we obtain the corresponding linearized equations involving only the $\mathrm{z}$ dependent parts of the perturbations to the temperature and the z-components of the velocity denoted by $T$ and $w$ respectively,

$\left[\left(D^{2}-a^{2}\right)^{2}-Q D^{2}\right] w-a^{2} R T=0$,

$\left(D^{2}-a^{2}\right) T+f(z) w=0$,

subjected to

$w=0$,

$D T=0$,

$D^{2} w+a M^{1 / 2} T=0$,

at the upper free surface $\mathrm{z}=1$, and

$w=0$,

$D^{2} w=0$,

$T=0$.

on the lower rigid boundary $z=0$. The operator $\mathrm{D}=d / d z$ denotes the differentiation with respect to the vertical coordinate $z$. The variables $w$ and $T$ denote respectively the vertical variation of the $z$-velocity, and temperature. To investigate the effect of the non-uniform temperature gradient on the Bénard-Marangoni convection, six types of basic temperature profile are chosen and these have been listed in Table $\mathbf{1}$ as model 1 to 6 .

\section{SOLUTION}

We use the single-term Rayleigh-Rits technique to find the critical eigenvalue. Multiplying equation (10) by $w$ and equation (11) by $T$, integrating the resulting equations by parts with respect to $z$ from 0 to 1 , using the boundary conditions (12) (17) and taking $w=A w_{1}, T=B T_{1}$ in which $A$ and $B$ are constants and $w_{1}$ and $T_{1}$ are trial functions, yields the following equation for the eigenvalue:

$M=-\frac{\left[\left\langle\left(D^{2} w_{1}\right)^{2}+\left(2 a^{2}+Q\right)\left(D w_{1}\right)^{2}+a^{4} w_{1}^{2}\right\rangle-\frac{\left.a^{2} R\left\langle w_{1} T_{1}\right\rangle f(z) w_{1} T_{1}\right\rangle}{\left\langle\left(D T_{1}\right)^{2}+a^{2} T_{1}^{2}\right\rangle}\right]\left\langle\left(D T_{1}\right)^{2}+a^{2} T_{1}^{2}\right\rangle}{a^{2} D w_{1}(1) T(1)\left\langle f(z) w_{1} T_{1}\right\rangle}$

In equation (18), $\langle.$.$\rangle denotes integration with respect to z$ between $z=0$ and $z=1$. We select the following trial functions

$w_{1}=z^{2}(1-z)(3-2 z), T_{1}=1$

such that they satisfy all the boundary conditions (12) - (17) except the one given by equation (14), $D^{2}{ }_{w}+a^{2} M T=0$ but the residual from this is included in the residual from the differential equations. Substituting equations (19) into (18), we get

$$
M=\frac{a^{2}\left(4536+19 a^{4}+432 a^{2}+16 Q\right)-630 a^{2} R\left(3 z^{2}-5 z^{3}+2 z^{4}\right\rangle\left\langle f(z)\left(\left\langle 3 z^{2}-5 z^{3}+2 z^{4}\right\rangle\right)\right\rangle}{\left.630 a^{2} f(z)\right)\left\langle 3 z^{2}-5 z^{3}+2 z^{4}\right\rangle}
$$

\section{RESULTS AND DISCUSSION}

In this paper, we study the onset of steady instability of the Bénard-Marangoni convection arising due to the buoyancy and surface tension forces in the presence of magnetic field with non-uniform basic temperature gradient. Our study here is to show that a suitable nonlinear temperature profile, magnetic field and buoyancy forces suppress or augment Marangoni convection which is important in the material processing in the laboratory. In each case investigated in this paper, we can identify the critical minima of the marginal stability curves in the $(a, M)$ plane. For a given set of parameters, the critical Marangoni number for the onset of convection is defined as the minimum of the global minima of marginal stability curve. We denote this critical value by $M_{c}$ and the corresponding critical wave number by $a_{c}$.

Table 2 shows the work of Nield [6] for the insulating case, $Q=0$ and $M=0$. As seen in Table 2, the agreement between the present results and the results from the literature (Nield [6]) is satisfactory for $Q=0$ and $M=0$. The critical Marangoni number $M_{c}$ and the corresponding wave number $a_{c}$ for different value of $Q$ and $R$ is presented in Table 3. We recover the results of Pearson [2] for $Q=0$ in the linear basic temperature case $f(z)=1$ with $R=0$. A comparison of the

Table 1. Reference Steady-State Temperature Gradient

\begin{tabular}{|c|c|c|}
\hline Model & Reference Steady-State Temperature Gradient & $f(z)$ \\
\hline 1 & Linear & 1 \\
\hline 2 & Inverted parabola & $2(1-z)$ \\
\hline 3 & Parabola & $2 z$ \\
\hline 4 & Step function & $\delta(z-\varepsilon)$, where $\delta$ is the Dirac delta-function. \\
\hline 6 & Piecewise linear (cooled from above) & $\begin{array}{l}0 \text { for } 0 \leq z<1-\varepsilon \\
\varepsilon^{-1} \text { for } 1-\varepsilon<z \leq 1\end{array}$ \\
\hline
\end{tabular}


critical Marangoni number in Table 3 shows that the step function (Model 4) is the most destabilizing basic temperature distribution, because the jump in temperature occurs nearer the free surface, and the inverted parabolic (Model 2) is the most stabilizing temperature distribution. From Table 3, it is observed that for the critical Marangoni number, the inequality holds for the six models

$$
M_{c 4}<M_{c 6}<M_{c 3}<M_{c 5}<M_{c 1}<M_{c 2}
$$

It can be seen from Table 3, the critical Marangoni number increases with increasing Chandrasekhar number $Q$. This is expected since the presence of the uniform magnetic field suppresses the Marangoni convection. Physically, the magnetic field gives rise to the Lorentz force. This force is in a direction opposite to the direction of velocity and hence makes the system more stable. Significant suppression of the convective flow can be obtained by applying strong magnetic field. Table $\mathbf{3}$ also shows that the value of the critical wave number for every temperature profiles remain the same for various values of the Chandrasekhar number. The presence of the magnetic field has no effect on the value of the critical wave number. Further inspection in Table $\mathbf{3}$ shows that the critical Marangoni number decreases with an increase of the Rayleigh number, for the assigned value of the Chandrasekhar number.. The corresponding effect of the increasing magnetic field on the Marangoni convection may be viewed in Fig. (1) for $R=0$. The figure indicates that the critical Marangoni number is a monotonic increasing function of Chandrasekhar number $Q$. It is expected that the imposition of the external magnetic field may play an important role in suppressing convective flow.

In the case of piecewise linear and step function profiles, the critical Marangoni number depends on the thermal depth $\varepsilon$. But, we found that the presence of magnetic field does not give the effect for critical thermal depth $\varepsilon_{c}$ for each of these 3 types of temperature gradient. Fig. (2) illustrates the critical Marangoni number $M_{c}$ as a function of thermal depth $\varepsilon$ for step function and piecewise linear profiles with selected values of $R$ and $Q$. This figure shows that for piecewise linear profile arising from heated from below, the critical Marangoni numbers decrease monotonically with an increase in the thermal depth. However, for step function and piecewise linear profile arising from cooled from above, the critical Marangoni numbers do not decrease monotonically with thermal depth. The critical Marangoni numbers first decrease, reach some minimum values, and then increase with increasing $\varepsilon$. From this figure, we note that when the magnetic field is imposed, the critical Marangoni number increases.

Table 2. Critical Rayleigh Number $\boldsymbol{R}_{c}$ for $Q=0$ and $M=0$

\begin{tabular}{|c|c|c|}
\hline $\boldsymbol{R}_{c}$ & Nield [6] & Present Study \\
\hline \hline$R_{c 4}$ & 184.6 & 184.6 \\
\hline$R_{c 6}$ & 252.0 & 252.0 \\
\hline$R_{c 3}$ & - & 288.0 \\
\hline$R_{c 5}$ & 292.5 & 292.6 \\
\hline$R_{c 1}$ & 320.0 & 320.0 \\
\hline$R_{c 2}$ & - & 360.0 \\
\hline
\end{tabular}

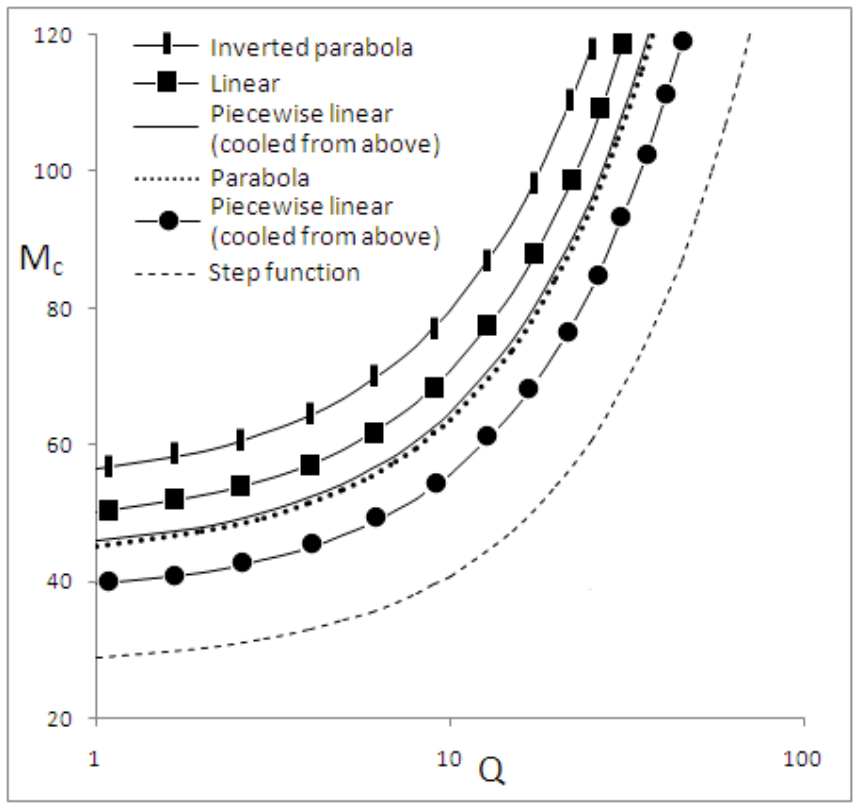

Fig. (1). Critical Marangoni number as a function of Chandrasekhar number for $R=0$.

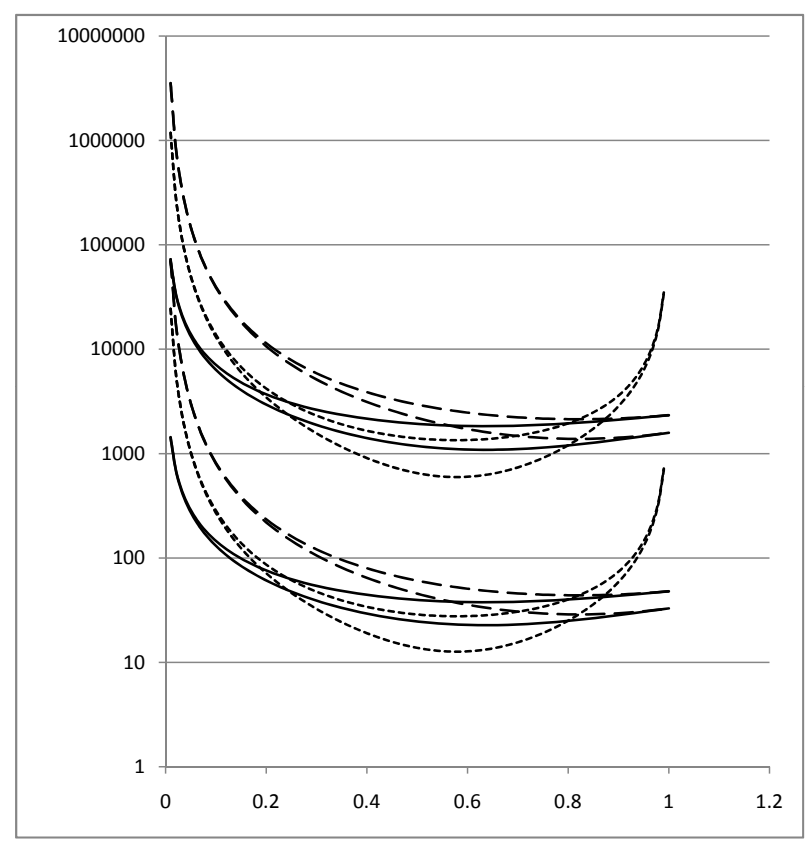

Fig. (2). Critical Marangoni number as a function of thermal depth for various values of $Q$ and $R$.

\section{CONCLUSIONS}

We have considered the influences of non-uniform basic temperature gradients and magnetic field on the onset of Bénard-Marangoni convection in a fluid layer with non-slip and insulated at the bottom. The single Galerkin procedure provides a good method for establishing this problem. We found that the step function is the most destabilizing basic temperature distribution, because the jump in temperature occurs nearer to the free surface. The critical Marangoni 
Table 3. Critical Marangoni number $M_{c}$ for Various Values of $Q$ and $R$

\begin{tabular}{|c|c|c|c|c|c|c|c|c|}
\hline$Q$ & $a_{c}$ & $\boldsymbol{R}$ & $M_{c 4}$ & $M_{c 6}$ & $\boldsymbol{M}_{c 3}$ & $M_{c 5}$ & $\boldsymbol{M}_{c 1}$ & $M_{c 2}$ \\
\hline 0 & 0 & $\begin{array}{l}0 \\
100.000 \\
184.634 \\
251.964 \\
288.000 \\
292.613 \\
320.000 \\
360.000\end{array}$ & $\begin{array}{l}27.695 \\
12.695 \\
0\end{array}$ & $\begin{array}{l}37.795 \\
22.795 \\
10.099 \\
0\end{array}$ & $\begin{array}{l}43.200 \\
28.200 \\
15.505 \\
5.405 \\
0\end{array}$ & $\begin{array}{l}43.892 \\
28.892 \\
16.197 \\
6.097 \\
0.692 \\
0\end{array}$ & $\begin{array}{l}48.000 \\
33.000 \\
20.305 \\
10.205 \\
4.800 \\
4.108 \\
0\end{array}$ & $\begin{array}{l}54.000 \\
39.000 \\
26.305 \\
16.205 \\
10.800 \\
10.108 \\
6.000 \\
0\end{array}$ \\
\hline 10 & 0 & $\begin{array}{l}0 \\
100.000 \\
272.555 \\
371.947 \\
425.143 \\
431.953 \\
472.381 \\
531.429\end{array}$ & $\begin{array}{l}40.883 \\
25.883 \\
0\end{array}$ & $\begin{array}{l}55.792 \\
40.792 \\
14.909 \\
0\end{array}$ & $\begin{array}{l}63.771 \\
48.771 \\
22.888 \\
7.979 \\
0\end{array}$ & $\begin{array}{l}64.793 \\
49.793 \\
23.910 \\
9.001 \\
1.022 \\
0\end{array}$ & $\begin{array}{l}70.857 \\
55.857 \\
29.974 \\
15.065 \\
7.086 \\
6.064 \\
0\end{array}$ & $\begin{array}{l}79.714 \\
64.714 \\
38.831 \\
23.922 \\
15.943 \\
14.921 \\
8.857 \\
0\end{array}$ \\
\hline 100 & 0 & $\begin{array}{l}0 \\
100.000 \\
1063.845 \\
1451.793 \\
1659.429 \\
1686.011 \\
1843.810 \\
2074.286\end{array}$ & $\begin{array}{l}159.577 \\
144.577 \\
0\end{array}$ & $\begin{array}{l}217.769 \\
202.769 \\
58.192 \\
0\end{array}$ & $\begin{array}{l}248.914 \\
233.914 \\
89.338 \\
31.145 \\
0\end{array}$ & $\begin{array}{l}252.902 \\
237.902 \\
93.325 \\
35.133 \\
3.987 \\
0\end{array}$ & $\begin{array}{l}276.571 \\
261.571 \\
116.995 \\
58.803 \\
27.657 \\
23.670 \\
0\end{array}$ & $\begin{array}{l}311.143 \\
296.143 \\
151.566 \\
93.374 \\
62.229 \\
58.241 \\
34.571 \\
0\end{array}$ \\
\hline
\end{tabular}

number increases with increasing Chandrasekhar number. One can conclude that the convective enhancement induced by buoyancy and surface tension forces can be suppressed when the external magnetic field is applied. The magnetic field and inverted parabolic temperature profile increase $M_{c}$ considerably. Hence, they make the system more stable than other cases. Therefore, we conclude that a suitable strength of magnetic field and inverted parabolic temperature profile is favourable for material processing in the laboratory with stimulated microgravity environment.

\section{ACKNOWLEDGEMENTS}

The authors gratefully acknowledged the financial support received in the form the Ministry of Science, Technology and Innovation, Malaysia (MOSTI).

\section{REFERENCES}

[1] L. Rayleigh, "On the convection currents in a horizontal layer of fluid when the higher temperature is on the other side", Phil. Mag., vol. 32, pp. 529-546, 1916.

[2] J. R. A. Pearson, "On convection cells induced by surface tension", J. Fluid Mech., vol. 4, pp. 489-500, 1958.

[3] D. A. Nield, "Surface tension and buoyancy effects in cellular convection", J. Fluid Mech., vol. 19, pp. 341-352, 1964.
[4] A. Vidal, and A. Acrivos, "Nature of the neutral state in surfacetension driven convection", Phys. Fluids, vol. 9, pp. 615-616, 1966.

[5] W. R. Debler, and L. F. Wolf, "The effects of gravity and surface tension gradients on cellular convection in fluid layers with parabolic temperature profiles", Trans. Am. Soc. Mech. Engrs, Series C. J. Heat Transfer, vol. 92, pp. 351-358, 1970.

[6] D. A. Nield, "The onset of transient convective instability", $J$ Fluid Mech., vol. 71, pp. 441-454, 1975.

[7] N. Rudraiah, "The onset of transient marangoni convection in a liquid layer subjected to rotation about a vertical Axis", Mater. Sci. Bull., Indian Acad. Sci., vol. 4, pp. 297-316, 1982.

[8] R. Friedrich, and N. Rudraiah, "Marangoni convection in a rotating fluid layer with non-uniform temperature gradient", Int. J. Heat Mass Transfer, vol. 27, pp. 443-449, 1984.

[9] G. Lebon, and A. Cloot, "Effects of non-uniform temperature gradients on bénard-marangoni's instability", J. Non-Equilib. Thermodyn., vol. 6, pp. 15-30, 1981.

[10] N. Rudraiah, and V. Ramachandramurthy, "Effects of non-uniform temperature gradient and coriolis force on bénard-marangoni's instability", Acta Mech., vol. 61, pp. 37-50, 1986.

[11] K. T. Chiang, "Effect of a non-uniform basic temperature gradient on the onset of bẻnard-marangoni convection: Stationary and oscillatory analyses", Int. Commun. Heat Mass Transfer, vol. 32, pp. 192-203, 2005.

[12] R. Idris, H. Othman, and I. Hashim, "On effect of non-uniform basic temperature gradient on bẻnard-marangoni convection in micropolar fluid", Int. Commun. Heat Mass Transfer, vol. 36, pp. 255-258, 2009.

[13] S. Chandrasekhar, Hydrodynamic and hydromagnetic stability, Oxford: Oxford University Press, 1961 
[14] D. A. Nield, "Surface tension and buoyancy effects in the cellular convection of an electrically conducting liquid in a magnetic field", Z. Angew. Math. Phys., vol. 17, pp. 131-139, 1966.

[15] N. Rudraiah, V. Ramachandramurthy, O. P. Chandna, "Effects of magnetic field and non-uniform temperature gradient on marangoni convection", Int. J. Heat Mass Transfer, vol. 28, pp. 1621-1624, 1985.
[16] N. Rudraiah, O.P. Chandna, and M. R. Garg, "Effect of nonuniform temperature gradient on magneto-convection driven by surface tension and buoyancy", Indian J. Tech., vol. 24, pp. 279284, 1986.

[17] M. I. Char, and C. C. Chen, "Effect of a non-uniform temperature gradient on the onset of oscillatory bẻnard-marangoni convection of an electrically conducting liquid in a magnetic field", Int. J. Engng. Sci., vol. 41, pp. 1711-1727, 2003.

(C) Isa et al.; Licensee Bentham Open.

This is an open access article licensed under the terms of the Creative Commons Attribution Non-Commercial License (http: //creativecommons.org/licenses/by$\mathrm{nc} / 3.0 /$ ) which permits unrestricted, non-commercial use, distribution and reproduction in any medium, provided the work is properly cited. 